\title{
Hypoxia accelerates intraplaque neovascularization derived from endothelial progenitor cells in carotid stenosis
}

\author{
Daina Kashiwazaki, MD, PhD, ${ }^{1}$ Masaki Koh, MD, ${ }^{1}$ Haruto Uchino, MD, PhD, ${ }^{1}$ Naoki Akioka, MD, ${ }^{1}$ \\ Naoya Kuwayama, MD, PhD, ${ }^{1}$ Kyo Noguchi, MD, PhD, ${ }^{2}$ and Satoshi Kuroda, MD, PhD1

\begin{abstract}
Departments of ${ }^{1}$ Neurosurgery and ${ }^{2}$ Radiology, Graduate School of Medicine and Pharmaceutical Sciences, University of Toyama, Japan
\end{abstract}

\begin{abstract}
OBJECTIVE The relationship between intraplaque hypoxia and intraplaque hemorrhage (IPH) has been reported, but the details remain obscure. In this study, the authors aimed to clarify the relationship among intraplaque hypoxia, endothelial progenitor cells (EPCs), and neovascularization, which causes IPH. The histological findings of specimens obtained from carotid endarterectomy were assessed.
\end{abstract}

METHODS This study included 49 patients who underwent carotid endarterectomy. Magnetic resonance plaque imaging was performed to analyze the components of the carotid plaques, and surgical specimens were subjected to immunohistochemical analysis. The numbers of hypoxia-inducible factor-1 alpha (HIF-1 $\alpha$ )-, CD34-, CD133-, and vascular endothelial growth factor receptor-2 (VEGFR-2)-positive cells in the carotid plaques were precisely quantified, as were the number and maximum diameter of CD31-positive microvessels.

RESULTS Plaque components were judged as fibrous in 7 samples, lipid-rich in 22, and IPH in 20. The number of CD34-, VEGFR-2-, and CD133-positive cells as an EPC-specific marker was significantly correlated with the number of HIF-1 $\alpha$-positive cells $(r=0.9, r=0.82$, and $r=0.81$, respectively). These numbers varied among the 3 plaque components (IPH > lipid-rich > fibrous). The number and maximum luminal diameter of CD31-positive microvessels were also significantly correlated with the number of HIF-1 $\alpha$-positive cells $(r=0.85$ and $r=0.89$, respectively) and varied among the 3 plaque components (IPH > lipid-rich > fibrous).

CONCLUSIONS The present findings suggest that intraplaque hypoxia may accelerate abnormal microvessel formation derived from EPCs, which in turn promotes IPH. The results also suggest that microvessel enlargement is a pivotal characteristic of IPH and these enlarged microvessels are immature endothelial tubes with disorganized branching and are fragile and prone to rupture.

https://thejns.org/doi/abs/10.3171/2018.4.JNS172876

KEYWORDS hypoxia; endothelial progenitor cell; carotid stenosis; plaque; vulnerability; vascular disorders

$\sim^{2}$ AROTID stenosis is associated with as many as $20 \%-25 \%$ of all ischemic strokes. ${ }^{21}$ Although over decade the degree of stenosis has routinely used as a measure of stroke risk, the character of plaques is garnering increasing interest as a risk factor for stroke. ${ }^{5}$ Plaques that contain a large, lipid-rich necrotic core, intraplaque hemorrhage (IPH), and inflammation are covered by a thin fibrous cap that is vulnerable to rup- ture. ${ }^{28}$ In particular, carotid plaque with IPH has been recognized as an indicator of a high risk for ischemic stroke, with accumulative evidence from histopathological and natural history studies using MR plaque imaging. ${ }^{11,23,31} \mathrm{Re}-$ cent reports have established a central role for microvessels and IPH in the initiation, progression, and rupture of atherosclerotic plaques. ${ }^{6}$ Furthermore, both microvessels and IPH contribute to inflammatory cell recruitment and

ABBREVIATIONS DAB = diaminobenzidine tetrahydrochloride; $\mathrm{EPC}=$ endothelial progenitor cell; $\mathrm{HIF}=$ hypoxia-inducible factor; $\mathrm{IPH}=$ intraplaque hemorrhage; $\mathrm{TOF}=$ time of flight; VEGF = vascular endothelial growth factor; VEGFR = VEGF receptor.

SUBMITTED November 14, 2017. ACCEPTED April 9, 2018.

INCLUDE WHEN CITING Published online October 5, 2018; DOI: 10.3171/2018.4.JNS172876. 
plaque instability. ${ }^{29}$ Thus, intraplaque microvessels might also be a marker of plaque vulnerability. ${ }^{6}$

The relationship between intraplaque hypoxia and plaque vulnerability has recently been reported. Some pathology studies suggested that the expression of hypoxiainducible factor-1 alpha (HIF-1 $\alpha$ ) and vascular endothelial growth factor (VEGF) is associated with IPH in carotid stenosis., ${ }^{917}$ However, the detailed pathological mechanisms through which microvessels and IPH develop remain obscure.

Endothelial progenitor cells (EPCs) are involved in neovascularization (CD31 positive) and endothelial integrity. There is growing evidence that some EPC subpopulations, characterized by surface markers such as CD34, VEGF receptor-2 (VEGFR-2), or CD133, might exert protective properties in arteriosclerosis. We previously reported that plaque microvessels play key roles in delivering EPCs from the peripheral blood to carotid plaques, which promotes plaque growth and the growth of microvessels. ${ }^{12}$ Recent studies have suggested that the overexpression of HIF-1 $\alpha$ enhances the proliferation, invasion, cell survival, and in vitro capillary sprout formation of the EPC population. ${ }^{24,32}$ In this study, therefore, we aimed to clarify the relation among intraplaque hypoxia, EPCs, and microvessels that cause IPH.

\section{Methods}

This study was approved by the IRB of our institution. Written informed consent was obtained from all patients. The study included a total of 49 patients, 45 men and 4 women, who underwent carotid endarterectomy at our hospital between July 2012 and November 2016. The mean patient age was $71.8 \pm 8.8$ years and ranged from 62 to 84 years. There were 43 symptomatic and 6 asymptomatic patients. Much of the following methods first appeared in our previous article. ${ }^{12}$

\section{MR Plaque Imaging}

In all patients, plaque components were evaluated using an MRI machine., $33,16,25$ For plaque characterization, long-axis and axial images of the carotid artery were obtained with 3D gradient-echo sequences in the area with the highest degree of stenosis. Three-dimensional timeof-flight (TOF) MR angiography was acquired through both carotid bifurcations in the axial plane. The imaging sequences were as follows: 1) 3D TOF: field of view $220 \mathrm{~mm} / 87.5 \%$, TR $23 \mathrm{msec}$, TE $7.00 \mathrm{msec}$; and 2) T1weighted: field of view $200 \mathrm{~mm} / 100 \%$, TR $500 \mathrm{msec}$, TE $11 \mathrm{msec}$. The slice thickness was $1.2 \mathrm{~mm}$ for $3 \mathrm{D}$ TOF and $2 \mathrm{~mm}$ for T1-weighted images.

The plaque component was defined according to signal intensity. Plaques with signal intensities $>200 \%$ of the signal intensity for muscle at any location or section were categorized as having high signal intensity; otherwise, plaques were categorized as having isointense signals. Plaques were considered fibrous when the signal intensity was isointense on both T1-weighted and TOF images. Plaques were considered lipid-rich when the signal intensity was high on T1-weighted images but isointense on TOF images. Plaques were considered to show IPH when the signal intensity was high on both T1-weighted and TOF images. The interval between MR plaque imaging was $<48$ hours for all patients. This method has been reported previously. ${ }^{13}$

\section{Immunohistochemical Analysis}

The carotid plaque specimens were fixed in $4 \%$ formaldehyde, embedded in paraffin, and cut into axial slices 4 $\mu \mathrm{m}$ thick. The section with the largest plaque burden was classified as the culprit lesion and subjected to staining. The deparaffinized sections were processed for antigen retrieval for 2 minutes in a pressure pot. Immunohistochemical analysis was used to identify the EPCs, hypoxic cells, and microvessels in the plaques. The expression levels of EPC-specific cell-surface markers, including CD34, VEGFR-2, and CD133, were confirmed with immunohistology. ${ }^{2,8,20}$ The expression levels of hypoxia cell markers, including HIF-1 $\alpha$ antibody, were confirmed with immunohistology. ${ }^{18}$ The expression levels of intraplaque microvessel markers, including CD31, were also confirmed with immunohistology. ${ }^{22}$

Briefly, each section was treated with the primary antibody against HIF-1 $\alpha$ (mouse monoclonal, 1:200 dilution, GT10211; Funakoshi), CD31 (mouse monoclonal, 1:200 dilution, TLD-3A12; Funakoshi), CD34 (mouse monoclonal, 1:100 dilution; BD Bioscience Pharmingen), VEGFR-2 (rabbit monoclonal, 1:100 dilution; Cell Signaling Technology, Inc.), and CD133 (rat monoclonal, 1:35 dilution; Abcam) for 40 minutes at $24^{\circ} \mathrm{C}$, followed by the Envision polymer of a DAKO EnVision+ Kit (DAKO Cytomation) for 60 minutes. The diaminobenzidine tetrahydrochloride (DAB) chromogen of a DAB substitute kit (DAKO Cytomation) was applied for 3-4 minutes, and hematoxylin was used for counterstaining.

Double-fluorescence immunohistochemistry was also used to confirm the types of cells present under hypoxic conditions in carotid plaques. Briefly, the sections were treated with monoclonal antibodies against CD34 (mouse monoclonal, 1:100 dilution; BD Bioscience Pharmingen) and HIF-1 $\alpha$ against CD34 at $25^{\circ} \mathrm{C}$ for 1 hour. Goat antimouse immunoglobulin $\mathrm{G}$ heavy and light secondary antibody (Alexa Fluor 555 conjugate) and donkey polyclonal secondary antibody to heavy and light rabbit immunoglobulin (Alexa Fluor 488) were added to identify cell immunoreactivities. The emitted fluorescence was observed through each appropriate filter using fluorescence microscopy and digitally photographed using a cooled chargecoupled device camera attached to the microscope (model BZ-9000; Keyence Co.).

The carotid plaque was divided into 4 lesions (shoulder, bottom, core, and interface-to-media region), as described previously. ${ }^{12}$ Shoulder lesions were defined as tissue adjacent to the outer border of the lipid core down to the vessel lumen. The counting of positive cells and microvessels was performed in shoulder lesions. The HIF-1 $\alpha$-positive cells, CD31-positive microvessels, and CD34-, CD133-, and VEGFR-2-positive cells were counted in each region using the cell counter tool of ImageJ software (National Institutes of Health). The positive cell counts and maximum microvessel luminal diameter measurements were performed by a certified neurosurgeon (D.K.). 

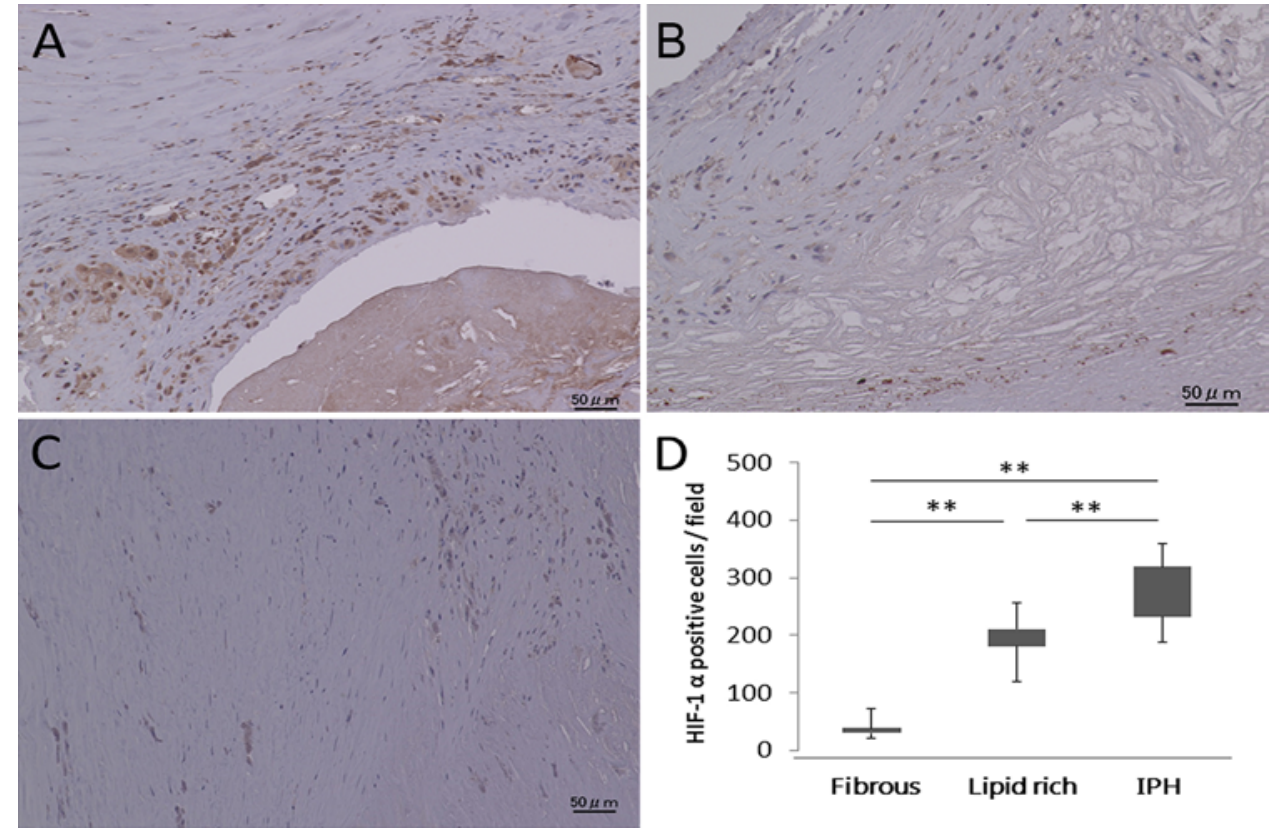

FIG. 1. Immunohistochemical analysis revealed that the number of HIF-1 $\alpha$-positive cells was higher in plaques with IPH (A) compared with lipid-rich plaques (B) and fibrous plaques (C). The number of HIF-1 $\alpha$-positive cells varied among the 3 plaque components and differed significantly (D). ${ }^{* *} p<0.01$, bar $=50 \mu \mathrm{m}$. Figure is available in color online only.

\section{Statistical Analysis}

Continuous data were statistically analyzed using 1-factor ANOVA followed by the post hoc Tukey-Kramer test, Pearson correlation coefficient, Spearman's rank correlation coefficient, or Mann-Whitney U-test, as appropriate. A $p$ value $<0.05$ was considered statistically significant.

\section{Results}

\section{Evaluation of Carotid Plaque Components With MR Plaque Imaging}

The results of preoperative MR plaque imaging yielded 7 fibrous plaques, 22 lipid-rich plaques, and 20 plaques with IPH. Therefore, there were 29 plaques without IPH and 20 plaques with IPH.

All 20 plaques with IPH and 18 of 22 lipid-rich plaques (81.8\%) had cap rupture or ulceration, which are markers of vulnerable plaque as operative findings. In contrast, none of 7 fibrous plaques had cap rupture or ulceration. The findings of MR plaque imaging were well correlated with operative findings.

\section{Relationship Between Intraplaque Hypoxia and EPCs in Carotid Plaques}

Immunohistochemical analysis showed that the total number of HIF- $1 \alpha$-positive cells widely varied among 3 plaque components: $39.5 \pm 19.8$ cells/field in fibrous plaques, $200.8 \pm 35.4$ cells/field in lipid-rich plaques, and $253.3 \pm 47.2$ cells/field in plaques with IPH. The differences in these counts reached statistical significance among the 3 plaque components (Fig. 1). The number of HIF$1 \alpha$-positive cells also significantly differed between the plaques with IPH and those without $(\mathrm{p}<0.01)$.

The counts of CD34-, VEGFR-2-, and CD133-positive cells in plaques with IPH were $251.1 \pm 68.1$ cells/field, 250.4 \pm 70.4 cells/field, and $101.0 \pm 20.3$ cells/field, respectively. The counts of CD34-, VEGFR-2-, and CD133-positive cells in lipid-rich plaques were $188 \pm 58.2$ cells/field, 169 \pm 60.4 cells/field, and $66.1 \pm 15.3$ cells/field, respectively. The counts of CD34-, VEGFR-2-, and CD133-positive cells in fibrous plaques were $70.3 \pm 28.2$ cells/field, 60.9 \pm 20.9 cells/field, and $24.1 \pm 10.3$ cells/field, respectively. The number of CD34-, VEGFR-2-, and CD133-positive cells in plaques varied among the 3 components. The number of HIF-1 $\alpha$-positive cells strongly correlated with the expression levels of EPC-specific markers, including CD34, VEGFR-2, and CD133 ( $\mathrm{r}=0.9, \mathrm{p}<0.01 ; \mathrm{r}=0.82$, $\mathrm{p}$ $<0.01$; and $\mathrm{r}=0.81, \mathrm{p}<0.01$, respectively; Fig. 2 ).

The number of CD31-positive microvessels in plaques varied among 3 plaque components: $40.1 \pm 19.1$ in fibrous plaques, $211.5 \pm 44.9$ in lipid-rich plaques, and $318.4 \pm$ 89.6 in plaques with IPH. The number of CD31-positive microvessels in plaques was strongly correlated with the number of HIF-1 $\alpha$-positive cells $(r=0.85, p=0.01$; Fig. 2 ). The results of double-fluorescence immunohistochemistry demonstrated that the CD34-positive cells were also positive for HIF-1 $\alpha$, which suggests that the CD34-positive cells were chronically exposed to hypoxia (Fig. 3).

\section{Enlarged Vessels, Hypoxia, and Plaque Components}

The maximum luminal diameter of microvessels in fibrous plaques, lipid-rich plaques, and plaques with IPH was $14.3 \pm 3.7 \mu \mathrm{m}$ (range 10.1-21.0 $\mu \mathrm{m}$ ), $35.7 \pm 9.6 \mu \mathrm{m}$ (range 20.1-54.3 $\mu \mathrm{m}$ ), and 59.5 $\pm 12.7 \mu \mathrm{m}$ (range 39.4-89.9 $\mu \mathrm{m})$, respectively. The maximal luminal diameter varied widely among the 3 plaque components, and the differences were statistically significant $(\mathrm{p}<0.01$; Fig. 4$)$. The difference in the maximal luminal diameter of microvessels 

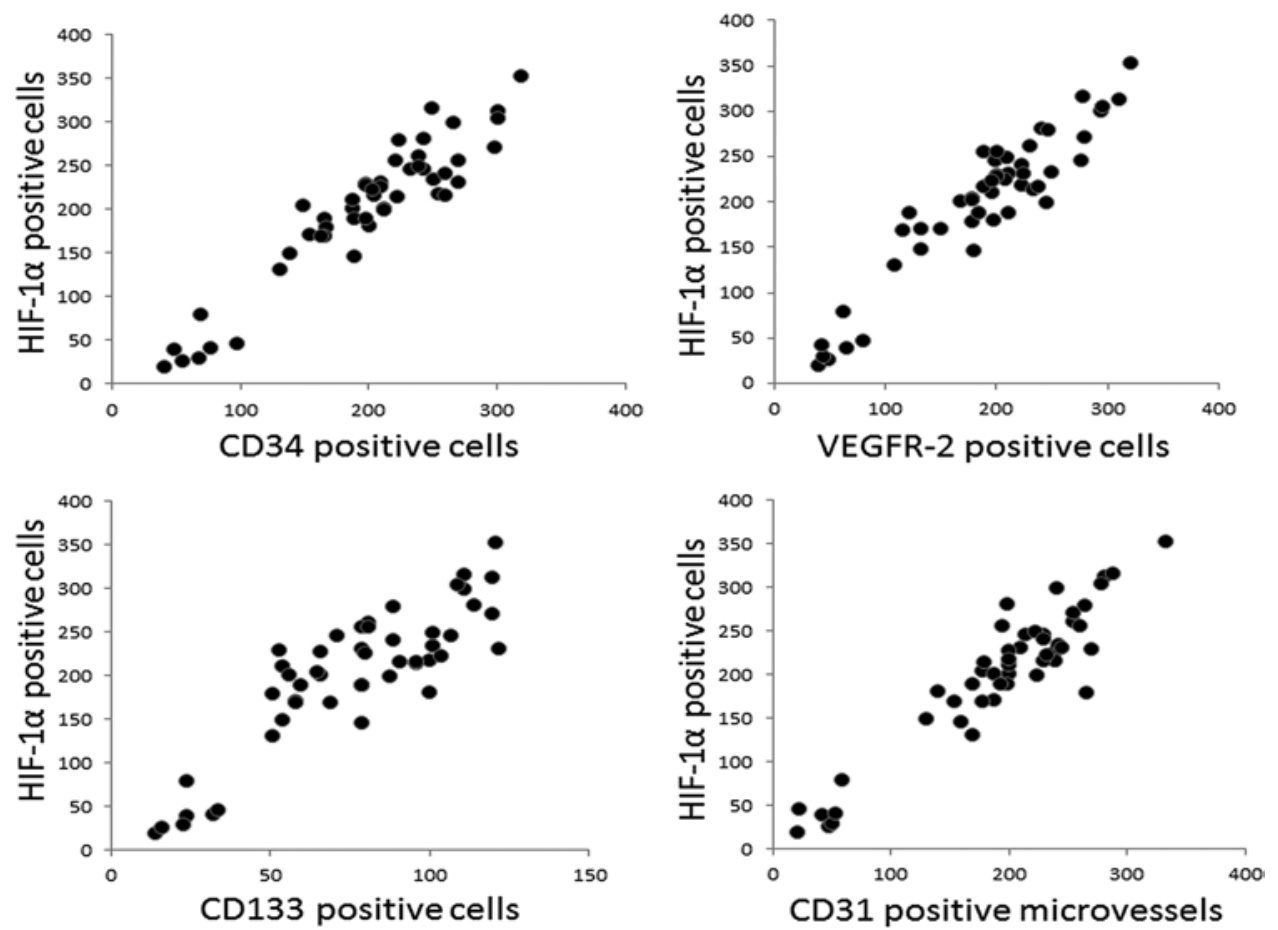

FIG. 2. Scatterplots demonstrate the significant correlations between HIF-1 $\alpha$ cells and CD34-, VEGFR-2-, and CD133-positive cells as well as CD31-positive microvessels $(r=0.9, p<0.01 ; r=0.82, p<0.01 ; r=0.81, p<0.01$; and $r=0.85, p<0.01$, respectively).

was also statistically significant between the plaques with IPH and those without $(\mathrm{p}<0.01)$. The threshold of maximal luminal diameter of microvessels between plaques with and without IPH was $42.0 \mu \mathrm{m}$ as analyzed with a receiver operating curve (sensitivity 0.83 , specificity 0.95 , area under the curve 0.96 , and $95 \%$ confidence interval $0.92-1)$. Therefore, the microvessels with a diameter $>42$ $\mu \mathrm{m}$ were considered at higher risk for IPH. The maximal luminal diameter of microvessels strongly correlated with
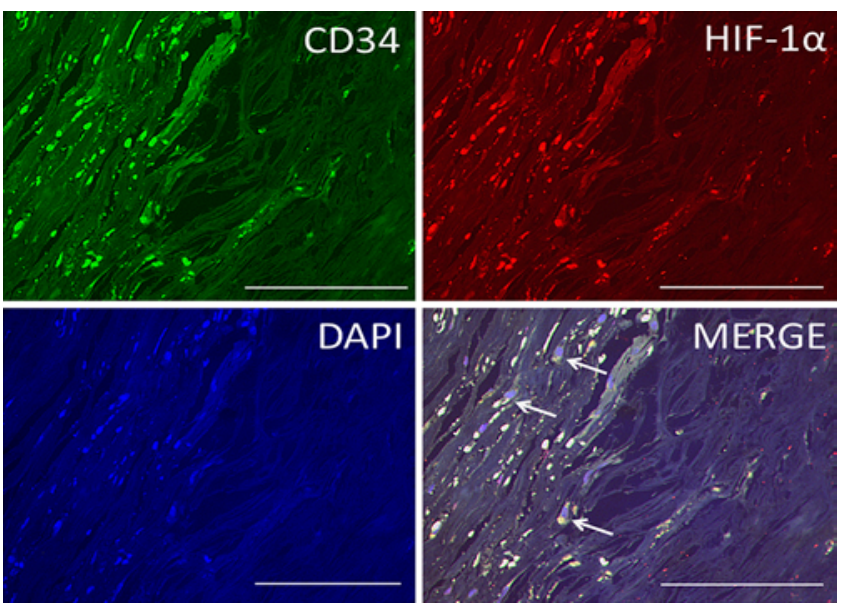

FIG. 3. The results of double-fluorescence immunohistochemistry demonstrated that CD34-positive cells (arrows) were also positive for HIF-1 $\alpha$, which suggests that the CD34-positive cells were hypoxic. Bar $=100 \mu \mathrm{m}$. DAPI = 4',6-diamidino-2-phenylindole. Figure is available in color online only. the number of HIF- $1 \alpha$-positive cells $(\mathrm{r}=0.89, \mathrm{p}<0.01$; Fig. 5). The walls of the enlarged microvessels $(>42 \mu \mathrm{m}$ in diameter) were strongly positive for VEGFR-2 (Fig. 5).

\section{Discussion}

The principal findings of this study are, first, that intraplaque hypoxia as indicated by the HIF-1 $\alpha$ expression is strongly correlated with the number of CD34-, VEGFR-2-, and CD133-positive cells. Compared with the fibrous plaques, plaques with IPH had higher expression levels of HIF-1 $\alpha$, CD34, VEGFR-2, and CD133. These data suggested that mobilization of circulating EPCs, which were induced by hypoxia of unstable plaque, accelerates the formation of microvessels derived from EPCs.

Second, the maximum luminal diameter of microvessels was correlated with the number of HIF-1 $\alpha$-positive cells. The diameter of enlarged vessels was larger in plaques with IPH compared with plaques without IPH. The threshold of maximum luminal diameter of microvessels between plaques with and without IPH was 42 $\mu \mathrm{m}$. The endothelial cells of enlarged microvessels $(>42$ $\mu \mathrm{m})$ showed strong positivity against VEGFR-2 antibody. Their microenvironment suggests that these enlarged microvessels are immature endothelial tubes with disorganized branching and are fragile and prone to rupture.

\section{Intraplaque Hypoxia Accelerates EPC-Derived Neovascularization}

We found that the counts of CD31-positive microvessels and CD34-, CD133-, and VEGFR-2-positive cells were highly correlated with the expression level of HIF- 

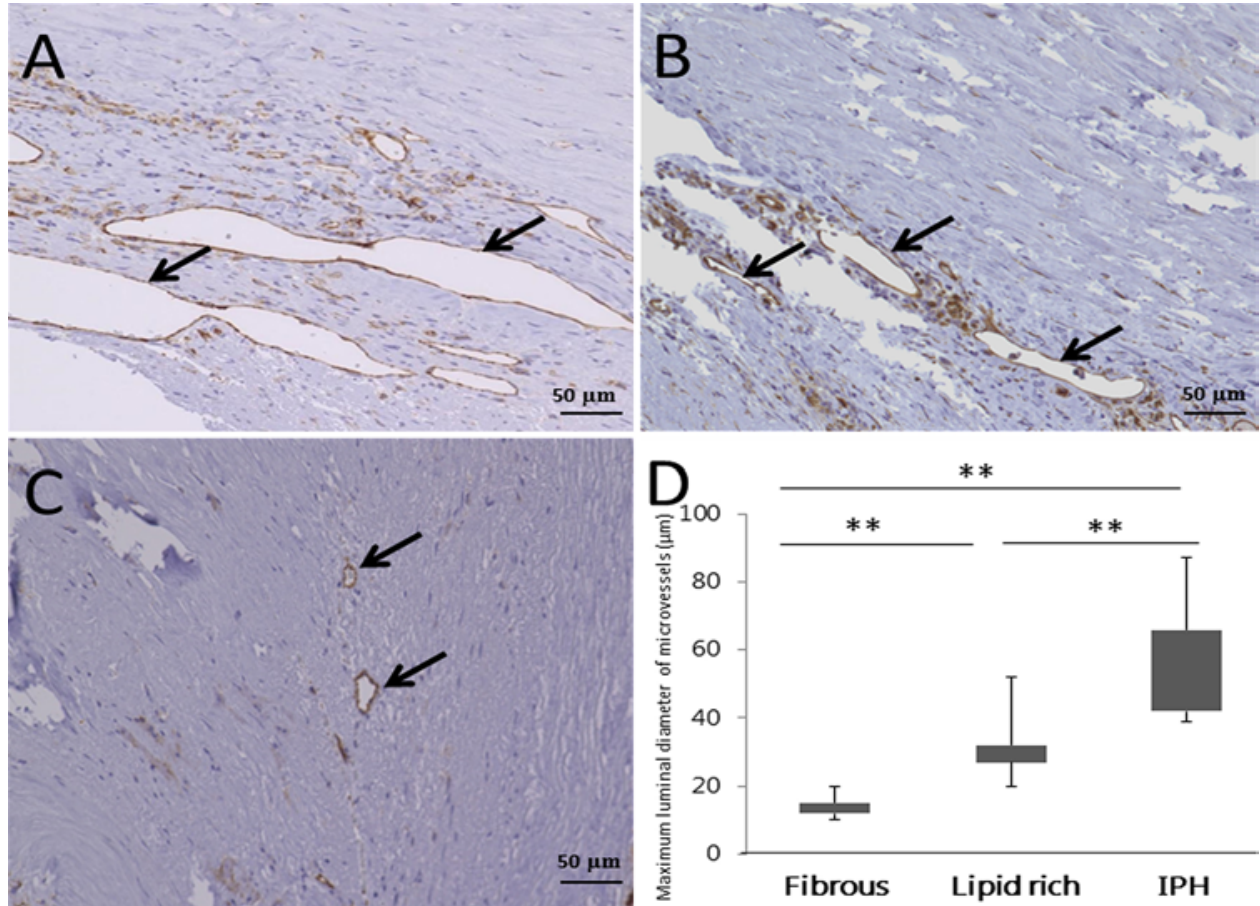

FIG. 4. Immunohistochemical analysis revealed that CD31-positive microvessels (arrows) in plaques with IPH (A) had diameters larger than those in lipid-rich plaques (B) and fibrous plaques (C). The diameter of CD31-positive vessels varied among the 3 plaque components and differed significantly (D). ${ }^{* *} p<0.01$. Figure is available in color online only.

1 $\alpha$. CD34, CD133, and VEGFR-2 are known as EPC markers. ${ }^{2,20,32}$ Larger numbers of CD31-positive microvessels, HIF-1 $\alpha$-positive cells, and EPCs were observed in plaques with IPH compared with fibrous and lipid-rich plaques. These results suggest that intraplaque hypoxia may play a pivotal role in the growth of microvessels derived from EPCs. Kütscher et al. suggested that HIF-1 $\alpha$ overexpression enhances the proliferation, invasion, cell survival, and in vitro capillary sprout formation of EPCs. ${ }^{15}$ In other studies, it was concluded that a combination of inflammation and accompanying intraplaque hypoxia is the stimulus for neovascularization. In rabbit aorta and human carotid arteries, the presence of hypoxia and nuclear accumulation of HIF-1 was demonstrated, in particular in
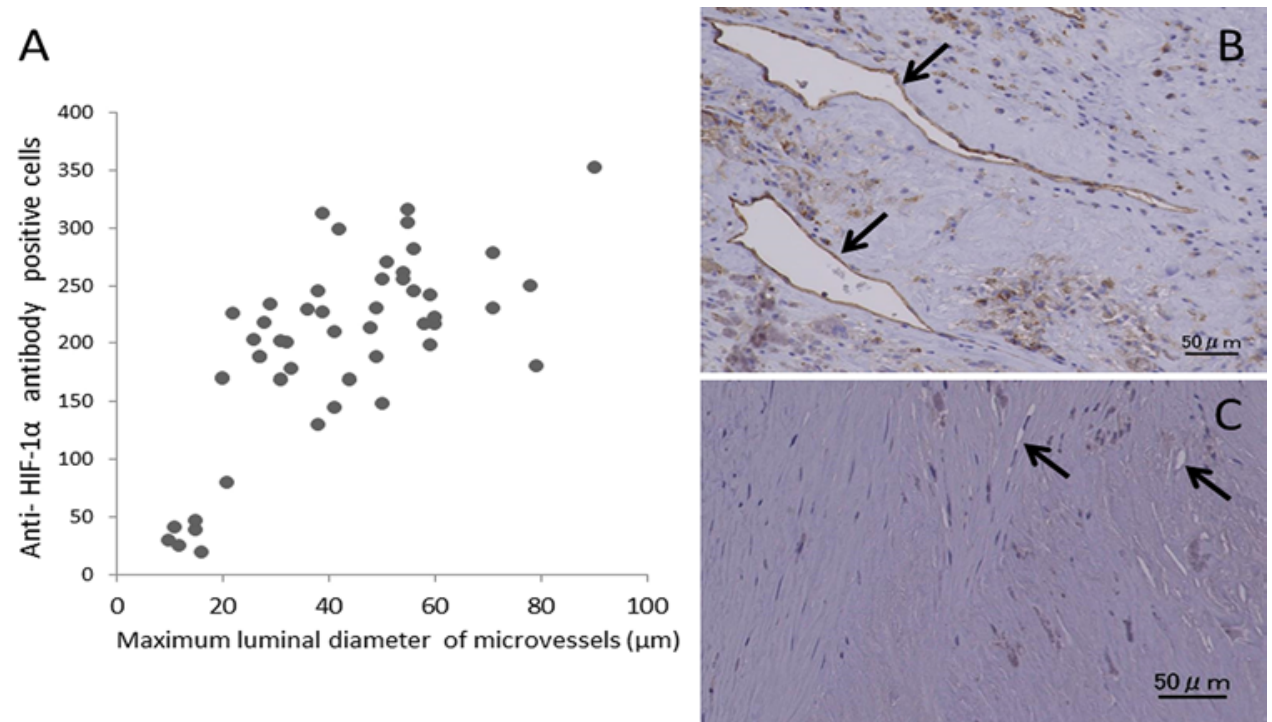

FIG. 5. A: A scatterplot demonstrates the significant correlations between HIF-1 $\alpha$-positive cells and the maximum diameter of CD31-positive vessels $(r=0.89, p<0.01)$. B: Immunohistochemical analysis showed that the endothelial cells of enlarged microvessels (arrows) were strongly positive against VEGFR-2 antibody. C: In contrast, endothelial cells of microvessels without enlargement (arrows) were negative against VEGFR-2 antibody. Figure is available in color online only. 

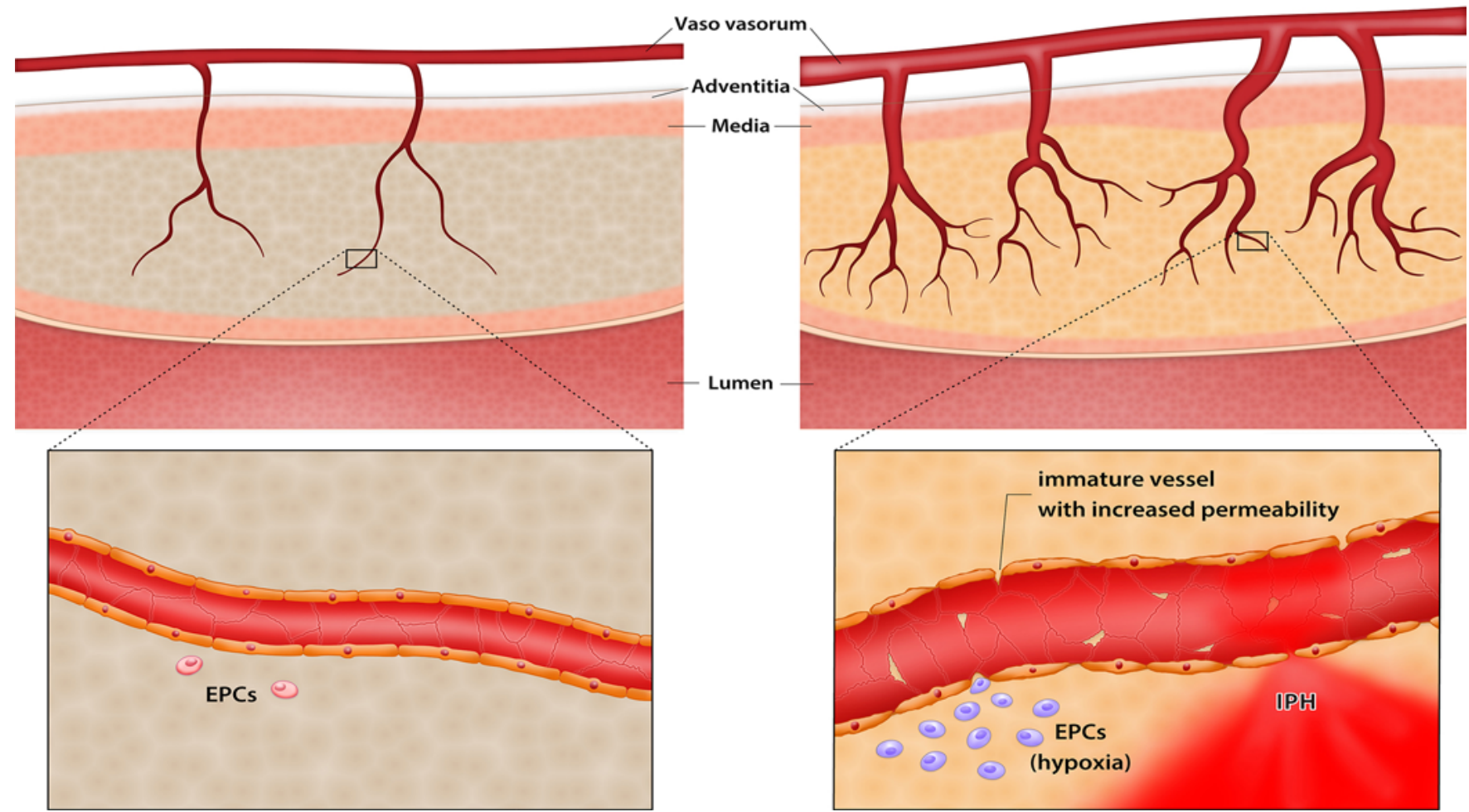

FIG. 6. A schematic representation of the key factors involved in plaque instability. Hypoxia accelerates microvessel formation derived from EPCs, and clinical features of unstable plaque, such as enlarged vessels with increased permeability, are prone to rupture. Figure is available in color online only.

the macrophage-rich center of the atherosclerotic lesion. This was accompanied by the enhanced expression of HIF-induced genes, the expression of angiogenic factors, neovascularization, and signs of ongoing inflammation. ${ }^{4,27}$ This mechanism supported our results, and further studies should be undertaken to explore it. Simons et al. reported an in vivo study showing that hypoxia, such as that resulting from ischemia-induced damage, induces the recruitment and migration of EPCs to hypoxic tissues ${ }^{24}$ Furthermore, Zhang et al. suggested the production of HIF-1 $\alpha$, induced by hypoxia, activated the apelin/expression of apelin receptor and downstream mitogen-activated protein kinase signaling pathways, thereby upregulating the proliferation of EPCs..$^{32}$ These studies support our results showing that hypoxia induces the activation of EPCs toward the growth of neovascularization in carotid plaques. Furthermore, the results of our double-fluorescence immunohistochemistry analysis consistently demonstrated that the CD34-positive cells were also positive for HIF-1 $\alpha$, which suggests that these cells were hypoxic. These findings were most remarkable in plaques with IPH. This study is the first to suggest a relationship between hypoxia and intraplaque microvessels derived from EPCs in human carotid stenosis. Our results may indicate that hypoxic CD34-positive cells are potential diagnostic and therapeutic targets in patients with carotid stenosis. Akhtar et al. suggested that endothelial HIF-1 $\alpha$ promoted atherosclerosis by triggering miR-19a-mediated CXCL1 expression and monocyte adhesion. ${ }^{1}$ Therefore HIF-1 $\alpha$ promotes inflammation and microvessel formation in atherosclerosis via several pathways. However, Chaudhari and colleagues reported that HIF-1 $\alpha$ plays a protective role in atherosclerosis. The increase of HIF-1 $\alpha$ in unstable plaque may be the result of a process of protective reaction. ${ }^{7}$

\section{Enlarged Microvessel Formation in Vulnerable Plaques}

Our results suggest that the maximum microvessel diameter is strongly correlated with IPH. Enlarged vessels $(>42 \mu \mathrm{m})$ were observed in IPH plaques. Furthermore, the maximum luminal diameter of microvessels was strongly correlated with hypoxia, which suggests that microvessel enlargement accelerated by hypoxia plays a pivotal role in promoting IPH. The formation of microvessels has been extensively researched and occurs in response to hypoxic environments. Furthermore, Kumamoto et al. suggested that the presence of microvessels within plaques is associated with rupture..$^{14}$ Evidence of the role of hypoxia in the initiation of angiogenesis stems from the demonstration of increased levels of HIF-1, which ultimately promotes VEGF production. ${ }^{4}$

VEGF gene expression is regulated by a variety of stimuli. Hypoxia is one of the most potent inducers of VEGF expression. ${ }^{30}$ Notably, excessive VEGF expression leads to vasodilation and an increase in vascular permeability. ${ }^{26} \mathrm{In}$ our study, the endothelial cells of enlarged vessels showed strong positivity against VEGFR-2 antibody. Their microenvironment suggested that these enlarged microvessels 
are immature endothelial tubes with disorganized branching and are fragile and prone to rupture. These microvessels are key regulators of plaque instability. Many previous reports have noted that vessel density and vessel number are important factors associated with IPH. ${ }^{10,17,19,23}$ The results of our study demonstrated that microvessel luminal enlargement is also a pivotal characteristic of IPH.

\section{Conclusions}

Our results suggested that intraplaque hypoxia accelerates microvessel formation derived from EPCs and that clinical features such as enlarged vessels are potential diagnostic and therapeutic targets in patients with carotid stenosis. Figure 6 is a schematic representation of the key factors involved in plaque instability.

Despite the limitations of this study, including the limited number of samples, small sample size of asymptomatic plaques, and its correlative nature, the study accurately reflects and encapsulates the immunohistological characteristics of carotid plaques. Future studies should include larger populations and focus on the therapeutic control of EPCs and hypoxia in patients with carotid plaque.

\section{Acknowledgments}

This study was funded by the Japan Society for the Promotion of Science Grants-in-Aid for Scientific Research (grant no. 16K10712).

\section{References}

1. Akhtar S, Hartmann P, Karshovska E, Rinderknecht FA, Subramanian $\mathrm{P}$, Gremse F, et al: Endothelial hypoxia-inducible factor-1 $\alpha$ promotes atherosclerosis and monocyte recruitment by upregulating microRNA-19a. Hypertension 66:12201226, 2015

2. Asahara T, Murohara T, Sullivan A, Silver M, van der Zee R, $\mathrm{Li} \mathrm{T}$, et al: Isolation of putative progenitor endothelial cells for angiogenesis. Science 275:964-967, 1997

3. Bitar R, Moody AR, Leung G, Symons S, Crisp S, Butany J, et al: In vivo 3D high-spatial-resolution MR imaging of intraplaque hemorrhage. Radiology 249:259-267, 2008

4. Björnheden T, Levin M, Evaldsson M, Wiklund O: Evidence of hypoxic areas within the arterial wall in vivo. Arterioscler Thromb Vasc Biol 19:870-876, 1999

5. Brinjikji W, Huston J III, Rabinstein AA, Kim GM, Lerman A, Lanzino G: Contemporary carotid imaging: from degree of stenosis to plaque vulnerability. J Neurosurg 124:27-42, 2016

6. Cattaneo M, Staub D, Porretta AP, Gallino JM, Santini P, Limoni C, et al: Contrast-enhanced ultrasound imaging of intraplaque neovascularization and its correlation to plaque echogenicity in human carotid arteries atherosclerosis. Int J Cardiol 223:917-922, 2016

7. Chaudhari SM, Sluimer JC, Koch M, Theelen TL, Manthey $\mathrm{HD}$, Busch M, et al: Deficiency of HIF1 $\alpha$ in antigen-presenting cells aggravates atherosclerosis and type $1 \mathrm{~T}$-helper cell responses in mice. Arterioscler Thromb Vasc Biol 35:2316-2325, 2015

8. Herrmann M, Binder A, Menzel U, Zeiter S, Alini M, Verrier S: CD34/CD133 enriched bone marrow progenitor cells promote neovascularization of tissue engineered constructs in vivo. Stem Cell Res (Amst) 13 (3 Pt A):465-477, 2014

9. Higashida T, Kanno H, Nakano M, Funakoshi K, Yamamoto I: Expression of hypoxia-inducible angiogenic proteins (hy- poxia-inducible factor-1 $\alpha$, vascular endothelial growth factor, and E26 transformation-specific-1) and plaque hemorrhage in human carotid atherosclerosis. J Neurosurg 109:83-91, 2008

10. Hiyama T, Tanaka T, Endo S, Komine K, Kudo T, Kobayashi $\mathrm{H}$, et al: Angiogenesis in atherosclerotic plaque obtained from carotid endarterectomy: association between symptomatology and plaque morphology. Neurol Med Chir (Tokyo) 50:1056-1061, 2010

11. Hosseini AA, Simpson RJ, Altaf N, Bath PM, MacSweeney ST, Auer DP: Magnetic resonance imaging plaque hemorrhage for risk stratification in carotid artery disease with moderate risk under current medical therapy. Stroke 48:678685,2017

12. Kashiwazaki D, Akioka N, Kuwayama N, Hayashi T, Noguchi K, Tanaka K, et al: Involvement of circulating endothelial progenitor cells in carotid plaque growth and vulnerability. J Neurosurg 125:1549-1556, 2016

13. Kashiwazaki D, Akioka N, Kuwayama N, Noguchi K, Tanaka K, Kuroda S: Pathophysiology of acute cerebrovascular syndrome in patients with carotid artery stenosis: a magnetic resonance imaging/single-photon emission computed tomography study. Neurosurgery 76:427-434, 2015

14. Kumamoto M, Nakashima Y, Sueishi K: Intimal neovascularization in human coronary atherosclerosis: its origin and pathophysiological significance. Hum Pathol 26:450-456, 1995

15. Kütscher C, Lampert FM, Kunze M, Markfeld-Erol F, Stark GB, Finkenzeller G: Overexpression of hypoxia-inducible factor-1 alpha improves vasculogenesis-related functions of endothelial progenitor cells. Microvasc Res 105:85-92, 2016

16. Moody AR, Murphy RE, Morgan PS, Martel AL, Delay GS, Allder S, et al: Characterization of complicated carotid plaque with magnetic resonance direct thrombus imaging in patients with cerebral ischemia. Circulation 107:3047-3052, 2003

17. Ogata A, Kawashima M, Wakamiya T, Nishihara M, Masuoka J, Nakahara Y, et al: Carotid artery stenosis with a highintensity signal plaque on time-of-flight magnetic resonance angiography and association with evidence of intraplaque hypoxia. J Neurosurg 126:1873-1878, 2017

18. Parathath S, Mick SL, Feig JE, Joaquin V, Grauer L, Habiel $\mathrm{DM}$, et al: Hypoxia is present in murine atherosclerotic plaques and has multiple adverse effects on macrophage lipid metabolism. Circ Res 109:1141-1152, 2011

19. Parma L, Baganha F, Quax PHA, de Vries MR: Plaque angiogenesis and intraplaque hemorrhage in atherosclerosis. Eur J Pharmacol 816:107-115, 2017

20. Peichev M, Naiyer AJ, Pereira D, Zhu Z, Lane WJ, Williams M, et al: Expression of VEGFR-2 and AC133 by circulating human $\mathrm{CD}^{+} 4^{+}$cells identifies a population of functional endothelial precursors. Blood 95:952-958, 2000

21. Petty GW, Brown RD Jr, Whisnant JP, Sicks JD, O'Fallon WM, Wiebers DO: Ischemic stroke subtypes: a populationbased study of incidence and risk factors. Stroke 30:25132516, 1999

22. Purushothaman M, Krishnan P, Purushothaman KR, Baber U, Tarricone A, Perez JS, et al: Genotype-dependent impairment of hemoglobin clearance increases oxidative and inflammatory response in human diabetic atherosclerosis. Arterioscler Thromb Vasc Biol 32:2769-2775, 2012

23. Qiao Y, Etesami M, Astor BC, Zeiler SR, Trout HH III, Wasserman BA: Carotid plaque neovascularization and hemorrhage detected by MR imaging are associated with recent cerebrovascular ischemic events. AJNR Am J Neuroradiol 33:755-760, 2012

24. Simons D, Grieb G, Hristov M, Pallua N, Weber C, Bernhagen J, et al: Hypoxia-induced endothelial secretion of macrophage migration inhibitory factor and role in endothelial 
progenitor cell recruitment. J Cell Mol Med 15:668-678, 2011

25. Singh N, Moody AR, Zhang B, Kaminski I, Kapur K, Chiu $\mathrm{S}$, et al: Age-specific sex differences in magnetic resonance imaging-depicted carotid intraplaque hemorrhage. Stroke 48:2129-2135, 2017

26. Sinnathamby T, Yun J, Clavet-Lanthier ME, Cheong C, Sirois MG: VEGF and angiopoietins promote inflammatory cell recruitment and mature blood vessel formation in murine sponge/Matrigel model. J Cell Biochem 116:45-57, 2015

27. Sluimer JC, Gasc JM, van Wanroij JL, Kisters N, Groeneweg M, Sollewijn Gelpke MD, et al: Hypoxia, hypoxia-inducible transcription factor, and macrophages in human atherosclerotic plaques are correlated with intraplaque angiogenesis. J Am Coll Cardiol 51:1258-1265, 2008

28. Underhill HR, Yuan C, Yarnykh VL, Chu B, Oikawa M, Dong L, et al: Predictors of surface disruption with MR imaging in asymptomatic carotid artery stenosis. AJNR Am J Neuroradiol 31:487-493, 2010

29. Virmani R, Kolodgie FD, Burke AP, Finn AV, Gold HK, Tulenko TN, et al: Atherosclerotic plaque progression and vulnerability to rupture: angiogenesis as a source of intraplaque hemorrhage. Arterioscler Thromb Vasc Biol 25:2054-2061, 2005

30. Williams PA, Stilhano RS, To VP, Tran L, Wong K, Silva EA: Hypoxia augments outgrowth endothelial cell (OEC) sprouting and directed migration in response to sphingosine1-phosphate (S1P). PLoS One 10:e0123437, 2015

31. Yamada K, Kawasaki M, Yoshimura S, Shirakawa M, Uchida
$\mathrm{K}$, Shindo S, et al: High-intensity signal in carotid plaque on routine 3D-TOF-MRA is a risk factor of ischemic stroke. Cerebrovasc Dis 41:13-18, 2016

32. Zhang J, Liu Q, Fang Z, Hu X, Huang F, Tang L, et al: Hypoxia induces the proliferation of endothelial progenitor cells via upregulation of Apelin/APLNR/MAPK signaling. Mol Med Rep 13:1801-1806, 2016

\section{Disclosures}

The authors report no conflict of interest concerning the materials or methods used in this study or the findings specified in this paper.

\section{Author Contributions}

Conception and design: Kashiwazaki. Acquisition of data: Kashiwazaki, Koh, Akioka, Kuwayama, Noguchi. Analysis and interpretation of data: Uchino. Drafting the article: Kashiwazaki, Kuroda. Critically revising the article: Kuroda. Statistical analysis: Kashiwazaki. Administrative/technical/material support: Kuroda. Study supervision: Kuroda.

\section{Correspondence}

Daina Kashiwazaki: Graduate School of Medicine and Pharmaceutical Sciences, University of Toyama, Toyama, Japan. dkashiwa@med.u-toyama.ac.jp. 